\title{
A Comparative Study on the Advantages of Using a Stapling Device in a Splenectomy for Patients with Traumatic Splenic Injury
}

\author{
Si Hyun Choi, Young Sun Yoo *, Seong Pyo Mun \\ Department of Surgery, School of Medicine, Chosun University, Gwangju, Korea
}

\section{Article history:}

Received: August 13, 2020

Revised: September 8, 2020

Accepted: September 9, 2020

\section{${ }^{*}$ Corresponding Author:}

Young Sun Yoo

Department of Surgery, School of Medicine,

Chosun University, 365 Pilmundaero,

Donggu, Gwangju 61453, Korea

E-mail: ysyoo@chosun.ac.kr

\section{ORCID}

Si Hyun Choi

https://orcid.org/0000-0002-8633-1614

Young Sun Yoo

https://orcid.org/0000-0002-0451-9788

Seong Pyo Mun

https://orcid.org/0000-0002-7067-4751

\begin{abstract}
Purpose: Surgery is prioritized for a splenic injury when the patient is hemodynamically unstable or the injury is severe and there is an increased risk of bleeding. This study aimed to examine the outcomes of splenectomies where a surgical stapling device was used to reduce operation time and rapidly control bleeding.

Methods: This retrospective study included 53 patients who underwent a splenectomy for traumatic splenic injury at Chosun University Hospital between 2012 and 2017. Clinical outcomes including operation time (duration), blood transfusion amount (number of units), length of hospital stay, length of intensive care unit stay, and mortality rate were compared between patients who received conventional ligation [conventional group (CG)] and patients who received a splenectomy where a surgical stapling device was used [stapling group (SG)].

Results: The SG showed an average operation time of 17 minutes less than the CG, although the reduction was not statistically significant. No significant differences in estimated blood loss and blood transfusion amount were determined between the 2 groups, although the SG received 1 more unit of red blood cells for transfusion in the 48-hour post-operative period compared with the CG. One case of pancreatic fistula as a postoperative complication was reported in the SG.
\end{abstract}

Conclusion: The use of a surgical stapling device in a splenectomy may be considered for a hemodynamically unstable patient with splenic injury which caused severe anatomical damage.

Keywords: spleen, splenectomy, surgical stapler, trauma

\section{Introduction}

The spleen is a solid organ which is susceptible to injury in the event of blunt abdominal trauma. Hemoperitoneum is commonly caused by splenic injury, and severe splenic injuries can result in hypovolemic shock or death. Surgery has been the typical treatment of choice for a splenic rupture caused by abdominal trauma. However, the danger of post splenectomy infection, nonoperative conservative treatments, and embolization may be considered first, before surgery for traumatic splenic injuries [1-3].

Nonoperative treatments are likely to fail for high American Association for the Surgery of Trauma (AAST) grade splenic injury in a hemodynamically unstable patient or in an injury that is highly prone to bleeding, and in these cases, surgery must be prioritized [1,3]. The World Society of Emergency Surgery (WSES) has recently classified a splenic injury accompanied by hemodynamic instability as a WSES Grade IV regardless of the severity of anatomical damage, and claimed that such an injury must be actively treated by surgical means [4].

Various methods of splenectomy have been used to preserve the spleen $[5,6]$. However, because approximately $60 \%$ of patients receiving a splenectomy are multiple trauma patients, a splenectomy is viewed as time consuming, and total splenectomy must be elected over spleen preservation when the patient is bleeding profusely [7].

Technological advances in surgical stapling devices have 
not only reduced the duration of operation time but also the rate of bleeding related complications in surgery [8]. Aside from conventional surgical methods, vascular ligation using a stapling device is also becoming popular in splenectomies and is expected to improve prognosis. In this study, we aimed to examine and compare the clinical outcome between conventional splenectomy and splenectomy using a stapling device performed for patients with traumatic splenic injuries.

\section{Materials and Methods}

This study included patients who underwent splenectomy at Chosun University Hospital between January 2012 and December 2017. Patients who underwent splenectomy due to internal conditions such as hereditary spherocytosis, and idiopathic thrombocytopenic purpura, or iatrogenic splenic injuries were excluded. Among 117 patients, 54 were included in the analysis.

\section{Patient selection}

Patients who were hemodynamically unstable at the time of hospital admission and presented with a splenic injury AAST Grade 3 or above on computed tomography (CT) scans were consulted by 2 trauma surgeons to decide whether surgery was needed. If deemed necessary, an emergency splenectomy was performed. Hemodynamic instability was defined as having a systolic blood pressure of $\leq 90 \mathrm{mmHg}$ despite resuscitation [9].

Patients were assigned to 1 of 2 groups for retrospective analysis: a conventional group (CG), in which the splenic hilum was managed by ligating the splenic artery and vein using a conventional method, and a stapling group (SG), in which the hilum was ligated and cut using a surgical stapling device (DST Series TA 30/60 mm, Covidien, Boulder, CO, USA).

\section{Splenectomy method}

In both groups, laparotomies were performed through a midline incision. After the avascular peritoneal attachment and ligament were dissected, the splenic artery and splenic vein were ligated at the splenic hilum. In the CG, a traditional double ligation method was performed which controls bleeding using a clamp, and performs ligation using nonabsorbable sutures. In the SG, a surgical stapling device was used which ligates the splenic artery, vein, and hilum at the same time, once the hilum is exposed. A trauma surgeon determined whether the use of the stapling device was necessary based on whether the patient had poor vital signs (and thus required a quick operation), and the estimated amount of blood loss (based on the severity of the splenic injury). The decision was made solely by the surgeon during the operation according to the clinical situation.

\section{Outcome evaluation}

Patient data was collected from medical records: demographics, injury mechanism, multiple organ injury, systolic blood pressure on admission, pulse rate, hemoglobin level, AAST splenic injury grade, time to operation, operation time, number of units of blood used during intraoperative transfusions and within 48 hours after surgery, length of intensive care unit (ICU) stay, total length of hospital stay, complications, and mortality rates. Time to operation was defined as the duration of time from arrival at the emergency room to the start of the operation. Operation time was defined as the amount of time spent during the splenectomy. In cases where other injured organs were treated during an operation, operation time included only the time spent on the splenectomy, which was identified from anesthesia records. It was difficult to determine the exact amount of blood loss that occurred during the splenectomy based on the anesthesia records for the entire operation. Thus, the records were used for a general understanding of the blood loss. The amount of preoperative and intraoperative units of red blood cell (RBC) used during the transfusion and the number of units of RBC transfused within 48 hours after surgery were separately investigated.

The primary outcomes of this study were the duration of operation time and the number of units used in perioperative RBC transfusions. The secondary outcomes were postoperative complications, the length of ICU and hospital stay, and mortality rates.

\section{Statistical analysis}

This study was approved by the Institutional Review Board of Chosun University Hospital (IRB no.: 2020-04-018-001). Statistical analyses were performed using IBM SPSS Version 25.0 (IBM Co., Armonk, NY, USA). Pearson's chi-square test and Student $\mathrm{t}$ test were used to compare variables between the 2 groups. A $p \leq 0.05$ was considered statistically significant.

\section{Results}

Hilar ligation was performed using a conventional method in the CG of 31 patients, and using a surgical stapling device (SG) in 23 patients (Table 1). The mean age of the patients in the CG and SG were 42.7 and 47.2 years, respectively. The percentage of males in the respective groups was $87.1 \%(n=27)$ and $82.6 \%$ $(n=19)$. Falls $(n=15)$ were the most common mechanism of traumatic splenic injuries, followed by motorcycle traffic accidents (TA; $n=14)$, pedestrian TA $(n=10)$, and car driver TA $(n=10)$. Of all patients, 9.3\% $(n=5)$ had a splenic injury only; the remaining $90.7 \%(n=49)$ had injury in the spleen and at least 1 other organ. Seven patients (12.9\%) had injuries 
Table 1. Patient demographics and injury parameters.

\begin{tabular}{|c|c|c|c|}
\hline Variable & $\begin{array}{l}\text { Conventional group } \\
\qquad(N=31)\end{array}$ & $\begin{array}{l}\text { Stapling group } \\
\qquad(N=23)\end{array}$ & $p$ \\
\hline Male sex & $27(87.1)$ & $19(82.6)$ & 0.350 \\
\hline Age $(y)$ & $42.71 \pm 23.12$ & $47.22 \pm 20.45$ & 0.357 \\
\hline Mechanism of injury & & & 0.332 \\
\hline TA, car driver & $4(12.9)$ & $6(26.0)$ & \\
\hline TA, bicycle & $1(3.2)$ & $0(0)$ & \\
\hline TA, motorcycle & $9(29.0)$ & $5(21.7)$ & \\
\hline TA, cultivator & $4(12.9)$ & $0(0)$ & \\
\hline $\mathrm{TA}$, pedestrian & $4(12.9)$ & $6(26.0)$ & \\
\hline Fall & $9(29.0)$ & $6(26.0)$ & \\
\hline Other organs with injury & & & 0.141 \\
\hline Spleen only & $3(9.7)$ & $2(8.6)$ & \\
\hline Ribs & $16(52.6)$ & $3(13.0)$ & \\
\hline Kidney & $1(3.2)$ & $1(4.3)$ & \\
\hline Liver & $2(6.5)$ & $4(17.3)$ & \\
\hline Kidney and ribs & $1(3.2)$ & $2(8.6)$ & \\
\hline Liver and ribs & $4(12.9)$ & $5(21.7)$ & \\
\hline Lung and ribs & $0(0)$ & $3(13.0)$ & \\
\hline$>3$ organs & $4(12.9)$ & $3(13.0)$ & \\
\hline Combined operations & & & 0.505 \\
\hline Nephrectomy & $1(3.2)$ & $2(8.6)$ & \\
\hline Small bowel resection & $2(6.5)$ & $1(4.3)$ & \\
\hline Rib fixation & $2(6.5)$ & $1(4.3)$ & \\
\hline Liver packing & $1(3.2)$ & $1(4.3)$ & \\
\hline Distal pancreatectomy & $0(0)$ & $2(8.6)$ & \\
\hline ISS & $20.1 \pm 9.25$ & $22.5 \pm 12.94$ & 0.367 \\
\hline
\end{tabular}

Data are presented as $n(\%)$ or means \pm SD.

$\mathrm{TA}=$ traffic accident; ISS = injury severity score.

Table 2. Initial physiological indicators and AAST spleen injury grade.

\begin{tabular}{lcc}
\hline Variable & $\begin{array}{c}\text { Conventional group } \\
(N=31)\end{array}$ & $\begin{array}{c}\text { Stapling group } \\
(N=23)\end{array}$ \\
\hline Systolic blood pressure $(\mathrm{mmHg})$ & $88.3 \pm 26.0$ & $85.4 \pm 32.03$ \\
Heart rate (beats/min) & $89.1 \pm 16.5$ & $96.4 \pm 20.28$ \\
Hemoglobin level (g/dL) & $9.4 \pm 2.13$ & $8.8 \pm 2.61$ \\
AAST spleen injury grade & & 0.181 \\
3 & $7(22.6)$ & $3(13.0)$ \\
4 & $14(45.2)$ & $10(43.5)$ \\
\hline
\end{tabular}

Data are presented as $n(\%)$ or means \pm SD.

AAST $=$ American Association for the Surgery of Trauma. 
in 3 or more organs. No significant differences in the injury mechanism or number of organs involved were determined between both groups. According to combined injuries, several kinds of operations, such as nephrectomy, small bowel resection, rib fixation, liver packing for damage control surgery and distal pancreatectomy were performed simultaneously. There was also no significant difference in the mean injury severity score between both groups $(20.1 \pm 9.25$ and $22.5 \pm$ 12.94 for the CG and SG, $p=0.367$ ).

Regarding the early clinical parameters of patients at the time of emergency room admission, no significant differences in systolic blood pressure, pulse rate, and hemoglobin levels were determined between both groups (Table 2).

The severity of splenic injuries was determined using CT scans and the AAST splenic injury scale (Table 2). All patients who underwent a splenectomy had splenic injuries AAST Grade 3 or above. In both groups, AAST Grade 4 splenic injuries were the most common injuries with 14 cases $(45.2 \%)$ in the CG and 10 cases $(43.5 \%)$ in the SG. AAST Grade 5 injuries were the 2nd most common, with 10 cases (32.3\%) in the CG and 9 cases (43.5\%) in the SG.

No significant differences in the time to operation and operation time were determined between the 2 groups. The mean operation time was 17 minutes less in the SG group compared with the CG, however this was not significant (Table 3 ).
While there were no significant differences in the estimated blood loss, and in the number of preoperative, intraoperative, and postoperative RBC units transfused between both groups, the mean number of preoperative, intraoperative, and postoperative units of RBC transfused was 1 unit higher in the SG.

No significant difference in the rate of postoperative complications was determined between the 2 groups (Table 3 ). Five patients who suffered from wound dehiscence recovered after wound repair without any problems. One patient in the SG reported having a postoperative pancreatic fistula 2 weeks after surgery (Figure 1). CT scan showed the perisplenic fluid collection which was suggestive of a pancreatic fistula. The patient was discharged without any problems after undergoing percutaneous drainage.

No significant differences in the length of ICU and hospital stays and overall mortality were determined between the groups (Table 3 ). The rate of hypovolemia related mortality was 3.2\% $(n=1)$ and $8.7 \%(n=2)$ in the CG and SG, respectively. These 3 hypovolemia related mortality was mortality within 24 hours of admission due to uncontrolled bleeding and coagulopathy. The causes of the other 5 mortality cases were sepsis and resultant multiple organ failure following prolonged ICU hospitalization.

Table 3. Patient clinical outcomes.

\begin{tabular}{|c|c|c|c|}
\hline Variable & $\begin{array}{l}\text { Conventional group } \\
\qquad(N=31)\end{array}$ & $\begin{array}{l}\text { Stapling group } \\
\qquad(N=23)\end{array}$ & $p$ \\
\hline \multicolumn{4}{|l|}{ Time interval (min) } \\
\hline Time to operation & $151.9 \pm 127.14$ & $149.3 \pm 98.03$ & 0.936 \\
\hline Operation time & $126.4 \pm 34.75$ & $109.1 \pm 42.03$ & 0.104 \\
\hline Estimated blood loss (mL) & $1,274.1 \pm 1,268.21$ & $1,279.51 \pm 897.54$ & 0.986 \\
\hline \multicolumn{4}{|l|}{ RBC transfusion (units) } \\
\hline Pre- and intraoperative & $4(0-10)$ & $4(0-18)$ & 0.280 \\
\hline Postoperative (48 h) & $2(0-7)$ & $3(0-12)$ & 0.077 \\
\hline \multicolumn{4}{|l|}{ Complication } \\
\hline Wound dehiscence & $3(9.6)$ & $2(8.6)$ & \\
\hline Pancreatic fistula & $0(0)$ & $1(4.3)$ & \\
\hline ICU stay (d) & $1(0-13)$ & $2(0-12)$ & 0.705 \\
\hline Hospital stay (d) & $12(1-35)$ & $10(2-42)$ & 0.715 \\
\hline Overall mortality & $3(9.7)$ & $5(21.7)$ & 0.264 \\
\hline Hypovolemia related mortality & $1(3.2)$ & $2(8.7)$ & 0.459 \\
\hline
\end{tabular}

Data are presented as $n(\%)$ or means \pm SD.

Values of RBC transfusion, ICU stay and hospital stay are presented as median and range. Time to operation was defined as the duration of time from arrival at the emergency room until the start of the operation. Operation time was the amount of time taken to perform the splenectomy. $\mathrm{RBC}=$ red blood cell; ICU = intensive care unit. 


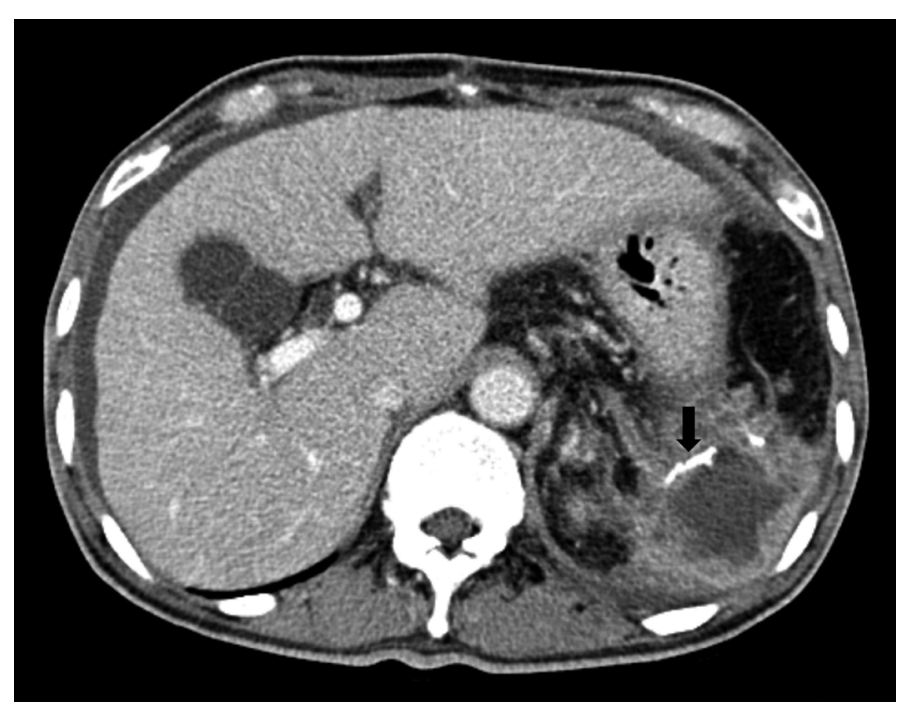

Figure 1. A computed tomography scan showing fluid collection in the perisplenic space after using a stapling device in the splenectomy which was suggestive of a pancreatic fistula (black arrow represents the staple lines).

\section{Discussion}

Surgical stapling devices have been consistently improved since their initial development by a Hungarian surgeon, Humer Hultl, in 1908 and are now widely used in gastrointestinal procedures and transection of vascular pedicles [10]. Stapling devices were initially employed to preserve the spleen in a partial splenectomy [11,12]. However, due to the highly vascular and friable anatomical characteristics of the parenchyma, bleeding control is likely to be difficult during a partial splenectomy for a traumatic splenic injury, necessitating a total splenectomy [13].

When ligating the splenic artery and vein by approaching the hilum in conventional splenectomy, blood vessels may not be easily exposed or bleeding may result during surgical manipulation, making the procedure time consuming. Vascular damage accompanying a splenic injury promotes even more bleeding, necessitating a greater amount of blood transfusion, operation time, and time required for anesthesia induction [14]. In the case of patients who have severe splenic injuries or are hemodynamically unstable due to multiple injuries, continuous bleeding into the peritoneum during splenic vessel ligation in a splenectomy may blur the surgery field of view, making it difficult to accurately identify anatomical structures.

The most troublesome step in a splenectomy is splenic hilum vessel ligation. En bloc stapling at the splenic hilum during ligation of the splenic artery and vein has been proposed as a means to overcome the difficulty of splenic hilum vessel ligation [15]. Since the introduction of attempts to perform a laparoscopic splenectomy for splenic injuries, using a stapling device on the splenic hilum it has become a standard approach, and has been reported as a safe and effective method that does not increase the rate of complications [16,17].

Operation time and intraoperative blood loss have been reported to affect the prognosis of patients undergoing a splenectomy $[18,19]$. Unnecessary dissection of the splenic hilum increases the operation time and intraoperative blood loss, which leads to greater transfusion requirements. As the amount of blood transfused increases, the risk of perioperative infections and multiple organ failure also increases; thus, an increased amount of blood transfusion is directly associated with poor postoperative outcomes [20]. Therefore, it is important to have a surgery strategy to reduce intraoperative transfusion requirements.

In this study, the trauma surgeon decided whether to use a surgical stapling device based on intraoperative vital signs and the severity of a splenic injury, and used the surgical stapling device during a splenectomy to reduce the operation time and transfusion requirements through quick bleeding control. In the SG, the operation time was approximately 17 minutes less than the $C G$, although the reduction was not statistically significant. In addition, the amount of blood used during transfusions, and the length of ICU and total hospital stays did not decrease in the SG. The SG also had a higher overall mortality rate than the CG (21.7\% vs. 9.7\%) although this difference was not significant. It is possible that the mortality rate appeared higher because patients in the SG presented with seemingly poorer vital signs and more severe splenic injuries than those in the CG.

Stapling devices allow for faster and easier vascular control via en bloc stapling of the hilum, but they can lead to complications such as bleeding, pancreatic fistulas, and arteriovenous fistulas, and should therefore be used with caution $[16,17]$. Although no bleeding related complications were reported in this study, there was 1 case of pancreatic fistula. Although pancreatic fistulas are reported to develop after a conventional splenectomy, caution is still needed to avoid damaging the tail of the pancreas by stapling close to the splenic hilum.

This study has a few limitations. Firstly, the data was retrospectively collected from medical records and were not randomized. Secondly, this study included a small population of patients who were admitted to a single hospital. Although patient demographics, injury parameters, and patient outcomes were not significantly different between the 2 groups, it is possible that patients with a clinically poor condition were included in the SG because the stapling device is typically used for severe injuries or injuries that involved a large amount of bleeding. A large scale, prospective, multicenter study is therefore needed to overcome these limitations. 


\section{Conclusion}

The use of a surgical stapling device during a splenectomy showed no significant difference in terms of the duration of operation time and requirements for blood transfusion compared with a conventional splenectomy.

In patients who are hemodynamically unstable or have a severe traumatic splenic injury that challenges splenic vessel ligation, the use of a stapling device may be considered to facilitate bleeding control. With careful manipulation of a stapling device to prevent postoperative complications such as pancreatic fistulas, splenectomies performed using a stapling device may become a viable treatment option for traumatic splenic injuries.

\section{Conflicts of Interest}

The authors have no conflicts of interest to declare.

\section{Funding}

This work was supported by grants from Chosun University Hospital, 2017.

\section{References}

[1] Watson GA, Rosengart MR, Zenati MS, Tsung A, Forsythe RM, Peitzman $A B$, et al. Nonoperative management of severe blunt splenic injury: are we getting better? J Trauma 2006;61(5):1113-8; discussion 1118-9.

[2] Requarth JA, D'Agostino RB Jr., Miller PR. Nonoperative management of adult blunt splenic injury with and without splenic artery embolotherapy: a meta-analysis. J Trauma 2011;71(4):898-903; discussion 903.

[3] Stassen NA, Bhullar I, Cheng JD, Crandall ML, Friese RS, Guillamondegui
OD, et al. Selective nonoperative management of blunt splenic injury: an Eastern Association for the Surgery of Trauma practice management guideline. J Trauma Acute Care Surg 2012;73(5 Suppl 4):S294-300.

[4] Coccolini F, Fugazzola P, Morganti L, Ceresoli M, Magnone S, Montori G, et al. The World Society of Emergency Surgery (WSES) spleen trauma classification: a useful tool in the management of splenic trauma. World J Emerg Surg 2019;14:30.

[5] Costamagna D, Rizzi S, Zampogna A, Alonzo A. Open partial splenectomy for trauma using GIA-Stapler and FloSeal matrix haemostatic agent. BM] Case Rep 2010;2010:bcr0120102601.

[6] Navas-Cuéllar JA, Cañete-Gómez J, López-Bernal F, García-Rivera C, ParejaCiuró F, Padillo-Ruiz J. Spleen-preserving surgery after blunt abdominal trauma with splenic hilum involvement. Cir Cir 2015;83(6):516-21. [in Spanish].

[7] Uranüs S, Kronberger L, Kraft-Kine J. Partial splenic resection using the TA-stapler. Am J Surg 1994;168(1):49-53.

[8] Chekan E, Whelan RL. Surgical stapling device-tissue interactions: what surgeons need to know to improve patient outcomes. Med Devices (Auckl) 2014;7:305-18.

[9] Mun SP, Yoo YS. Diversity of the Definition of Stable Vital Sign in Trauma Patients: Results of a Nationwide Survey. J Trauma Inj 2014;27(4):115-25.

[10] Gaidry AD, Tremblay L, Nakayama D, Ignacio RC, Jr. The History of Surgical Staplers: A Combination of Hungarian, Russian, and American Innovation. Am Surg 2019;85(6):563-6.

[11] Raschbaum G, Harnar TJ, Canizaro PC. The use of a stapler in splenic salvage as an alternative to the sutured partial splenectomy or splenorrhaphy. Surg Gynecol Obstet 1988;166(2):179-80.

[12] Ravo B, Ger R. Splenic preservation with the use of a stapling instrument: a preliminary communication. J Trauma 1988;28(1):115-7.

[13] Schroeppel TJ, Croce MA. Diagnosis and management of blunt abdominal solid organ injury. Curr Opin Crit Care 2007;13(4):399-404.

[14] Rhodes CA, Dinan D, Jafri SZ, Howells G, McCarroll K. Clinical outcome of active extravasation in splenic trauma. Emerg Radiol 2005;11(6):348-52.

[15] Demetriades D, Inaba K, Velmahos G. Atlas of Surgical Techniques in Trauma. Cambridge (UK): Cambridge University Press; 2015. p. 234-43.

[16] Vargün R, Göllü G, Fitöz S, Yagmurlu A. En-bloc stapling of the splenic hilum in laparoscopic splenectomy. Minim Invasive Ther Allied Technol 2007;16(6):360-2.

[17] Vecchio R, Marchese S, Swehli E, Intagliata E. Splenic Hilum Management During Laparoscopic Splenectomy. J Laparoendosc Adv Surg Tech A 2011;21(8):717-20.

[18] Timerbulatov MV, Senderovich EI, Gololobov Iu N. Laparoscopic splenectomy in a general surgery department of a hospital. Khirurgiia (Mosk) 2004(3):42-6. [in Russian].

[19] Amirkazem VS, Malihe K. Randomized clinical trial of ligasure ${ }^{\mathrm{TM}}$ versus conventional splenectomy for injured spleen in blunt abdominal trauma. Int J Surg 2017;38:48-51.

[20] Malone DL, Dunne J, Tracy JK, Putnam AT, Scalea TM, Napolitano LM. Blood transfusion, independent of shock severity, is associated with worse outcome in trauma. J Trauma 2003;54(5):898-905; discussion 905- 